\title{
Detection of the onset of upper-limb movements based on the combined analysis of changes in the sensorimotor rhythms and slow cortical potentials
}

J. Ibáñez ${ }^{1}$, J.I. Serrano ${ }^{1}$, M.D. del Castillo ${ }^{1}$, E. Monge ${ }^{2}$, F. Molina $^{2}$, I. Alguacil ${ }^{2}$, J.L. Pons ${ }^{1}$

1 Bioengineering Group, Spanish Research Council (CSIC), Arganda del Rey, Madrid E-28500

2 LAMBECOM, Universidad Rey Juan Carlos, Alcorcón, Madrid, Spain

E-mail: jaime.ibanez@csic.es 


\begin{abstract}
Background. Characterizing the intention to move by means of electroencephalographic activity can be used in rehabilitation protocols with patients' cortical activity taking an active role during the intervention. In such applications, the reliability of the intention estimation is critical both in terms of specificity -number of misclassifications- and temporal accuracy. Here, a detector of the onset of voluntary upper-limb reaching movements based on the cortical rhythms and the slow cortical potentials is proposed. The improvement in detections due to the combination of these two cortical patterns is also studied. Methods. Upper-limb movements and cortical activity were recorded in healthy subjects and stroke patients performing self-paced reaching movements. A logistic regression combined the output of two classifiers: i) a naïve Bayes classifier trained to detect the event-related desynchronization preceding the movement onset and ii) a matched filter detecting the bereitschaftspotential. The proposed detector was compared to the detectors using each one of these cortical patterns separately. In addition, differences between the patients and healthy subjects were analysed. Results. On average, $74.5 \pm 13.8 \%$ and $82.2 \pm 10.4 \%$ of the movements were detected with $1.32 \pm 0.87$ and $1.50 \pm 1.09$ false detections generated per minute in the healthy subjects and the patients, respectively. A significantly better performance was achieved by the combined detector (as compared to the detectors of the two cortical patterns separately) in terms of true detections $(p=0.099)$ and false positives $(p=0.0083)$. Conclusions. It is provided a rationale to combine information from cortical rhythms and slow cortical potentials to detect the onsets of voluntary upper-limb movements. It is demonstrated that the two cortical processes supply complementary information that can be summed up to boost the performance of the detector. Successful results have been also obtained with stroke patients, which supports the use of the proposed system in brain-computer interface applications with this group of patients.
\end{abstract}

\title{
1. Introduction
}

The use of electroencephalographic (EEG) activity to study cortical processes associated with the execution of movements has been explored widely [1,2,2-4]. The online decoding of this information has been successfully used to control external devices assisting patients with motor disabilities [5-8].

During the last years, the development of brain-computer interfaces (BCIs) for the functional rehabilitation of patients with motor disabilities has gained special interest $[9,10]$. The main purpose of BCIs in such scenarios is to provide a way to promote the neural rehabilitation of the patients. EEG-based systems allow the realtime characterization of cortical activity over the motor cortex while the subject is performing motor tasks, which allows the online detection when a person is attempting or imaging a movement [11-13], and to predict certain properties of the movement to be performed [14-17]. Such information may in turn be used to close the loop with neuroprosthetic or neurorobotic devices, thus resulting in a natural interface between the patient's expectations of movement and the actuation of external devices [18]. Recent studies have proven the importance of the proprioceptive feedback timing to achieve associative neural facilitation $[19,20]$. 
In a series of previous studies, use of the Bereitschaftspotential (BP) to detect the movement intention $[13,17,21-23]$ has been proposed. The BP is defined as a slow decay of the EEG voltage over the central regions of the cortex right before a voluntary movement is performed $[24,25]$. Because the BP is an identifiable pattern that is decaying until the movement starts, it is suitable to achieve temporal precision in the detection of voluntary movement onsets. In fact, previous studies showing results of online systems based on this pattern indicate that average latencies of $315 \pm 165$ ms can be obtained [23]. Nevertheless, the BP is not detectable in all cases, since some subjects do not present a significant pattern during self-paced movements. In addition, results obtained in previous studies using the BP have not fully validated the use of this cortical pattern alone to detect movement intentions in stroke patients [13]. In fact, altered BP patterns have been observed in previous studies with this type of patients $[26,27]$.

A possible way of boosting EEG-based systems aimed to detect the onsets of voluntary movements is to combine the BP with other EEG movement-related patterns providing complementary information [28]. The event-related desynchronization (ERD) is a well-known cortical pattern related to the execution of voluntary movements. The ERD over the sensorimotor cortex refers to the decrease of EEG signal power in the contralateral alpha $(8-12 \mathrm{~Hz})$ and beta $(13-30 \mathrm{~Hz})$ rhythms starting about $2 \mathrm{~s}$ before the onset of voluntary movements $[1,3,4,29]$. Although a variable anticipation may be observed in the ERD of a specific channel and frequency in a subject during consecutive movements, the spatio-tempo-frequential distribution of the ERD observed when averaging a number of EEG segments preceding voluntary movements shows a desynchronization pattern attached to the movement event [30]. Therefore, the analysis of the ERD also provides certain degree of information regarding the timing of volitional motor actions. Indeed, previous studies have used the ERD pattern to anticipate movement events $[12,31]$. As in the analysis of the BP, the ERD pattern of stroke patients presents variations with respect to healthy subjects [32]. Therefore, it is of special relevance to study how stroke-related cortical changes may affect a BCI driven by these cortical patterns.

This study presents for the first time a classifier combining the information obtained from the analysis of the BP and ERD cortical processes to estimate the onsets of voluntary upper-limb reaching movements. The system validation is performed using data from healthy subjects and chronic stroke patients. The comparison between the classifier combining the ERD and BP patterns and equivalent classifiers using either the BP or the ERD is also performed. Finally, the study describes the main differences found between patients and healthy subjects, which leads to a discussion on how EEG-based systems should be developed for rehabilitation applications. 


\section{Methods}

\subsection{Participants}

Two groups of subjects were recruited to validate the proposed system and also to find differences between the two groups. On the one hand, six healthy subjects (all males, right-handed and under 35 years old) were measured and considered the control group. On the other hand, the patients group was comprised of eight chronic stroke patients (three females, age $65 \pm 12$ years, mean $\pm \mathrm{SD}$; details are provided in Table 1 ). The present study shows results corresponding to all control subjects and patients P1-P6. Patients P7 and P8 were discarded for further analysis because they could not comply with the demands of the task performed during the experimental protocol. None of the subjects measured had prior experience with BCI paradigms.

\begin{tabular}{|c|c|c|c|c|c|c|c|c|c|}
\hline $\begin{array}{l}\text { Pat. } \\
\text { code }\end{array}$ & Age & Gender & $\begin{array}{l}\text { Stroke } \\
\text { type }\end{array}$ & $\begin{array}{c}\text { Affected } \\
\text { hemisphere }\end{array}$ & $\begin{array}{c}\text { Years since } \\
\text { stroke }\end{array}$ & Fügl-Meyer & Minimental & Ashworth & $\begin{array}{l}\mathrm{Rh} \text { sessions } \\
\text { a week }\end{array}$ \\
\hline $\mathrm{P} 1$ & 52 & $\mathrm{~F}$ & Isquemic & $\mathrm{L}$ & 4 & 126 & 30 & 0 & 1 \\
\hline P2 & 54 & M & Isquemic & $\mathrm{R}$ & 4 & 69 & 30 & 2 & 2 \\
\hline P3 & 54 & M & Isquemic & $\mathrm{L}$ & 3 & 68 & 30 & 3 & 2 \\
\hline $\mathrm{P} 4$ & 75 & M & Hemorrg & $\mathrm{L}$ & 1 & 60 & 30 & 3 & 2 \\
\hline P5 & 69 & M & Hemorrg & $\mathrm{R}$ & 4 & 64 & 29 & 3 & - \\
\hline P6 & 57 & $\mathrm{~F}$ & Isquemic & $\mathrm{L}$ & 1 & 93 & 26 & 1 & Discont \\
\hline P7 & 83 & $\mathrm{~F}$ & Isquemic & $\mathrm{L}$ & 5 & 112 & 23 & 1 & 2 \\
\hline P8 & 75 & M & Isquemic & $\mathrm{L}$ & 3 & - (mixed aphasia) & - & 2 & 2 \\
\hline
\end{tabular}

Table 1. Description of the patients participating in the present study

The experimental protocol was approved by the Ethical Committee of the Universidad Rey Juan Carlos (Madrid), and warranted its accordance with the Declaration of Helsinki. All participants signed a written informed consent.

\subsection{Experimental protocol}

The design of the experimental sessions was inspired in related studies [13]. Each participant was measured during one single session. The study was performed in a sound and light-attenuated room. Participants sat in a comfortable chair with their arms supported on a table. During the measurement phase, participants were instructed to remain relaxed with their eyes open and their gaze fixated on a point on the wall. They were asked to perform self-initiated reaching movements with the affected arm (the dominant arm in the case of the control subjects). The point to be reached was in the midline of the body and at $75 \%$ of the maximum distance achievable by each subject. The average distance between consecutive movements was around 8-15 s. During the resting state between movements, participants were asked to remain as relaxed and quite as possible, whereas they were asked to start a movement as soon as they felt the urge to do so.

Intervals containing at least $5 \mathrm{~s}$ of resting activity followed by a self-initiated reaching movement were considered trials and were used in the subsequent steps of 
the data analysis. On average, $53 \pm 8$ and $55 \pm 12$ trials were collected with the healthy subjects and the patients, respectively.

\subsection{Data Acquisition}

Arm movements were measured with solid-state gyroscopes and surface electromyography (sEMG). Three gyroscopes (Technaid S.L., Madrid, Spain), placed on the hand dorsum, the distal third of the forearm, and the middle of the arm measured the limb kinematics. The data were sampled at $100 \mathrm{~Hz}$.

Surface EMG was recorded using bipolar derivations on the main muscle groups involved in the execution of the reaching task (pectoralis major, anterior deltoids, medium deltoids, biceps, triceps and wrist extensors). The data were amplified (Zerowire Wireless EMG, Aurion, Milan, Italy) and sampled at 2,000 Hz.

EEG signals were recorded from 31 positions (AFz, F3, F1, Fz, F2, F4, FC3, FC1, FCz, FC2, FC4, C5, C3, C1, Cz, C2, C4, C6, CP3, CP1, CPz, CP2, CP4, P3, P1, Pz, P2, P4, PO3, PO4 and Oz, all according to the international 10-20 system) using active $\mathrm{Ag} / \mathrm{AgCl}$ electrodes (Acticap, Brain Products $\mathrm{GmbH}$, Germany). The reference was set to the voltage of the earlobe contralateral to the arm moved. AFz was used as ground. The signal was amplified (gUSBamp, g.Tecgmbh, Austria) and sampled at $256 \mathrm{~Hz}$.

All data were synchronised with a common digital signal and analysed offline using Matlab (The Mathworks Inc., Natick MA, USA).

\subsection{Detection of the onset of the movements}

To detect the time at which each movement started, kinematic information (gyroscopes) was used instead of the muscle activation data (sEMG). This decision was made to solve the difficulties in detecting muscle activation onset in spastic muscles likely found in the affected limbs of stroke patients.

The gyroscopic sensor that first detected that a movement was starting was used to locate the onsets of the reaching movements. This sensor selection was performed for each participant. Data were low-pass filtered (Butterworth, 2nd order, fc $=6 \mathrm{~Hz}$ ), and the peak amplitude was estimated for each subject performing the reaching movement. The threshold amplitude for detecting the onsets of the movements was set to $5 \%$ of this peak amplitude.

The sEMG data was used to ascertain that no sudden muscle activations were present in any of the muscles of the measured arm during the resting intervals between consecutive movements. Sudden muscle contractions (which were only observed in the patients in few moments along the recordings, such as when they readjusted their position on the chair to be comfortable) were marked as artifacts and were not considered in subsequent analyses. 


\subsection{Description of the classifier architecture and validation}

The following sections describe the design of two movement onset detectors, the first based on the characterization of the ERD preceding movement onset and the second characterizing the $\mathrm{BP}$ pattern. The process for combining the outputs of these two classifiers is described as well. The results section focuses mainly on the performance of the combined (ERD and BP) detector, although a comparison between the three proposed systems (based only on the BP, based only on the ERD and based on both processes) will also be addressed.

Since a limited number of trials could be acquired with certain subjects, the system was validated according to a leave-one-out methodology, i.e. once all trials had been identified, each of them was classified with a detector trained with the rest of the trials of the same session. The techniques applied here are specifically designed for real-time functioning of the proposed system. Therefore the results obtained with this validation methodology are expected to be similar to those obtained in a truly online scenario. After testing the system online, we decided to generate estimations of movement every $125 \mathrm{~ms}$ to enable the correct function (without data loss) of an average computer.

\subsubsection{ERD-based detector of the onset of the movement}

A naïve Bayes classifier was used to detect the ERD pattern preceding the movements. Previous studies have demonstrated the suitability of this type of classifiers for ERD detection [31,33]. Band-pass filtering was first applied to the EEG signals (Butterworth IIR filter, 3th order, $6 \mathrm{~Hz}<\mathrm{f} 1,35 \mathrm{~Hz}>\mathrm{f} 2$ ) and then a small laplacian filter (for each electrode position, the average voltage of the closest neighbours is subtracted) was used [34]. Frontal, fronto-central, central, centro-parietal and parietal channels were kept. The power values were estimated in segments of $1.5 \mathrm{~s}$ and for frequencies between 7-30 $\mathrm{Hz}$ in steps of $1 \mathrm{~Hz}$. Welch's method was used to this end (Hamming windows of $1 \mathrm{~s}, 50 \%$ overlapping). Estimations were generated every $125 \mathrm{~ms}$.

The power estimations obtained in all training trials from $-3 \mathrm{~s}$ to $-0.5 \mathrm{~s}$ (with respect to the movement onsets) were labelled as examples of the resting state, whereas the estimations generated at $\mathrm{t}=0 \mathrm{~s}$ where labelled as movement onset examples. The Bhattacharyya distance was used to select the 10 best features (channel/frequency pairs) to build the classifier. This number of features was chosen on the one hand to correctly characterize the ERD pattern in several channels and frequencies and, on the other hand, to achieve a real-time working classifier without requiring an excessively high computational load.

The trained classifier was applied to the test data generating estimations of movement intention every $125 \mathrm{~ms}$.

\subsubsection{BP-based detector of the onset of the movement}

A similar procedure to the one proposed in $[13,17]$ was used to detect the BP. However, unlike in those two previous studies, we used a finite impulse response band- 
pass filter with linear phase (FIR filter, 15th order, $0.05 \mathrm{~Hz}<\mathrm{f} 1,1 \mathrm{~Hz}>\mathrm{f} 2$ ) using the fir1 routine of Matlab software. This solution was adopted because linear preservation is crucial to extract the entire BP pattern, and using non linear phase filters (as for example the Butterworth filter) does not allow decoding of this pattern unless zerophase filtering (filtering in the forward and reverse direction) combined with framing of the EEG signal is performed, which leads to delayed (by a few hundreds of milliseconds) detection of the BP in the online function, due to filtering edge effects.

After the temporal filters were applied, spatial filtering and channel selection were performed. Three virtual channels were computed from the original 31 channels in the experimental set-up. These channels were obtained by subtracting the average potential of channels F3, Fz, F4, C3, C4, P3, Pz, and P4 from channels C1, Cz and C2 (similarly to [17]). The average BP was computed for the three resulting channels by using the training data. The channel showing the highest absolute peak at the movement onset was selected for the BP-based detection of movement onsets. We selected one of these channels instead of directly choosing $\mathrm{Cz}$ (as in [17]) because in healthy subjects, upper limb movements typically present a maximal late BP over the contralateral central areas of the cortex [25].

A matched filter was designed by using the previously selected channel. To this end, the average BP was obtained by using the time intervals from $-1.5 \mathrm{~s}$ to $0 \mathrm{~s}$ of the trials in the training dataset. The matched filter was applied to the virtual channel in the validation dataset. Estimations based on the test data were also made every 125 ms.

2.5.3. Detector of the movement onsets based on the combination of the ERD-and BP-based detectors

Outputs from ERD-based and BP-based detectors were combined by using a logistic regression classifier. Training examples of the resting condition were taken from estimations of the two detectors between $-3 \mathrm{~s}$ and $-0.5 \mathrm{~s}$ with respect to the movement onset (in steps of $125 \mathrm{~ms}$ ). The output estimations of the ERD and the BP classifiers at the movement onset were used to model the movement state. The classifier generated estimations of the intention to move every $125 \mathrm{~ms}$.

\subsection{Threshold selection}

On the test data, a threshold was applied to the output of the detector to decide at each moment whether movement intention was detected. The threshold was optimally obtained from the training dataset, following the criterion of maximizing the percentage of good trials (GT), i.e., trials with a true positive (TP) and with no false positives $(\mathrm{FP})$. The metrics are further defined in 2.7 . 


\subsection{Metrics of the detector performance and threshold selection}

Because the present study uses an asynchronous paradigm, conventional metrics used in traditional BCI paradigms could not be used $[35,36]$. Three metrics were used to evaluate the ability of the detector to reliably locate movement onsets. The TP rate was defined as the percentage of trials with movement detection contained in the time interval from $-0.75 \mathrm{~s}$ to $+0.75 \mathrm{~s}$ with respect to the actual onset estimated by the gyroscopes. The precision of the detector was characterized as the number of FP per minute $(\mathrm{FP} / \mathrm{min})$, i.e. rate of detections during the resting intervals. One or more false activations could be generated in a single trial. Therefore, the percentage of GT was obtained by counting the amount of trials in which no FP were generated and a TP was achieved. Finally, the latencies of the TP with respect to the actual onsets of movements were also computed to analyse the temporal accuracy of the system.

\subsection{Statistical analysis}

A comparison between the performance of the proposed detector combining the ERD and the BP information and the performances of detectors based only on each one of the two patterns was carried out to validate the proposed methodology. Given that the performances of the three detectors depend on each subject, a Friedman's test was used. To gain statistical power and reduce the size of the statistical results, samples from healthy subjects and patients were used together to test the hypothesis that the three proposed detectors supplied significantly different results. Bonferroni post-hoc correction was used to analyse significant differences between pairs. The statistical analysis was performed on the dependent variables GT, TP and FP/min. The rest of the presented results are reported as the mean $\pm \mathrm{SD}$ and are provided separately for patients and healthy subjects.

\section{Results}

\subsection{Summary of observed cortical patterns in patients and healthy subjects}

A summary of the average BP and ERD patterns observed in all patients and healthy subjects is shown in Figs. 1 and 2. Overall, the ERD and BP could be observed in most subjects analysed, although differences in spatial distribution and in strength of these patterns were found. The average BP peak across healthy subjects was found at $-19.8 \pm 57.6 \mathrm{~ms}$ with respect to movement onsets. In the case of the patients, this peak was observed at $97.5 \pm 47.3 \mathrm{~ms}$. A more homogeneous BP pattern could be observed in the group of healthy subjects than with the patients according to both, the temporal BP pattern and its spatial distribution. The ERD spatial distribution presented a predominant contralateral activation both in the alpha and beta bands in the group of healthy subjects, whereas activation patterns presented a central (P1, P2, and P5 in the alpha band and P2, P3, and P5 in the beta band) or bilateral distribution (P3 in the alpha band and $\mathrm{P} 1$ in the beta band) in the patients group. 

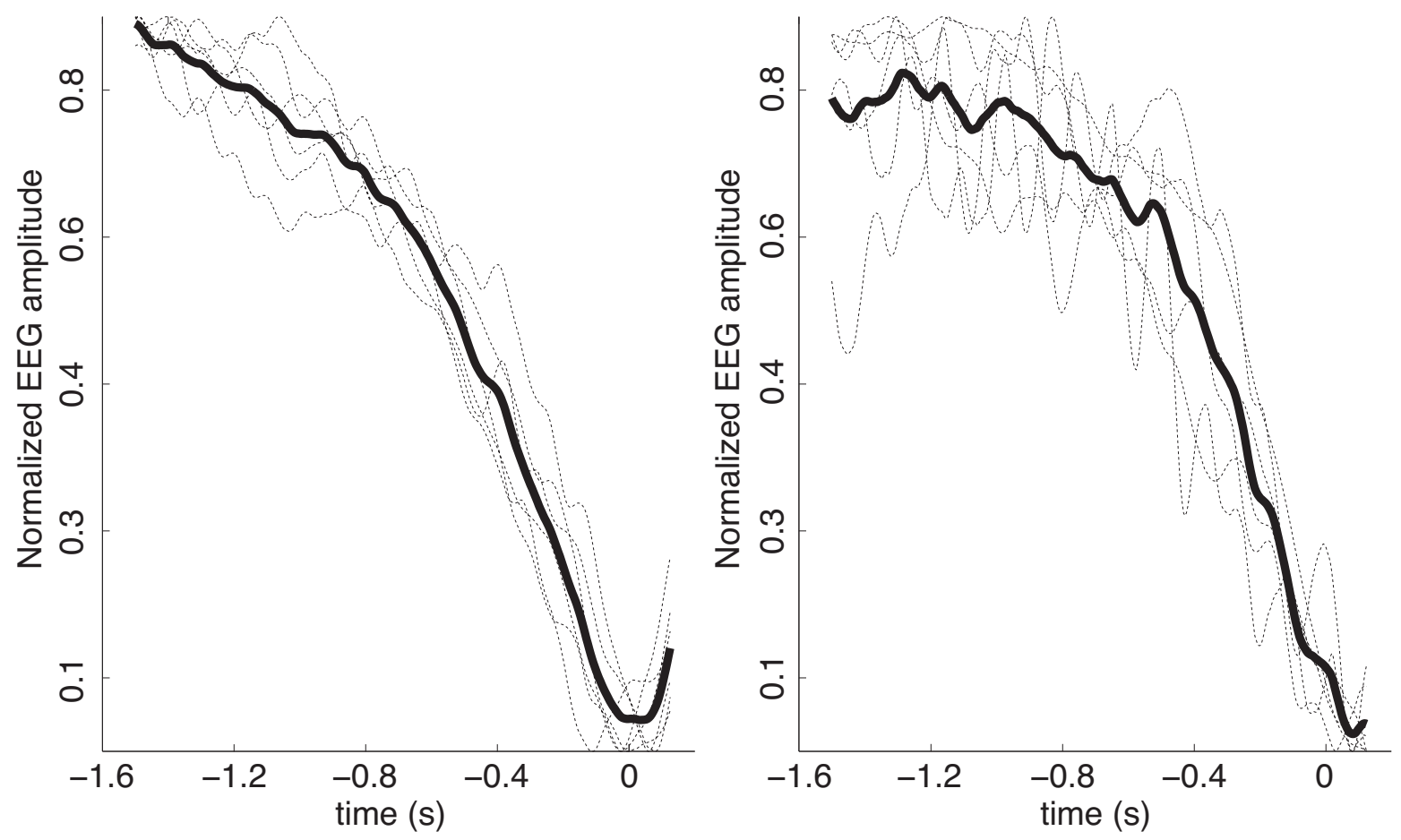

Figure 1. Average BP of all subjects (discontinuous lines), and average BP across subjects (solid line). Averages from healthy subjects and patients are presented in the left and right panels, respectively

\subsection{Results of the detection of the movement onsets}

Fig. 3 shows a representative example of the detector function on a single trial performed by participant $\mathrm{C} 2$. The different stages in EEG signal processing to extract information regarding movement intention are represented. The three last curves show the estimations of the onset of the movement based either on the BP pattern, on the ERD pattern, or on the combination of both, respectively. In this example, EEG-based detection is achieved with few hundreds of milliseconds of anticipation.

Table 2 summarizes the results obtained by the detector based on the ERD and BP patterns. On average $63.3 \pm 13.8 \%$ and $66.4 \pm 18.8 \%$ of GT are obtained with the healthy subjects and the patients, respectively. The percentage of true positives achieved with patients is smaller than with healthy subjects, but also the $\mathrm{FP} /$ min generated with the patients is higher. These results lead to a similar average performance of the system in terms of detections and false activations in both groups. Nevertheless, more delayed detections are obtained with patients $(35.9 \pm 352.3 \mathrm{~ms})$ than with healthy subjects $(-89.9 \pm 349.2 \mathrm{~ms})$.

The features selected by the ERD-based detector of movement onsets in the healthy subjects and patients are summarized in Table 3 and Table 4, respectively. According to the average ERD patterns observed in section 3.1 a predominance of contralateral central features is observed in the first case (healthy subjects), therefore most features correspond to channel $\mathrm{C} 3$ and the surrounding positions. In the case of the patients, 


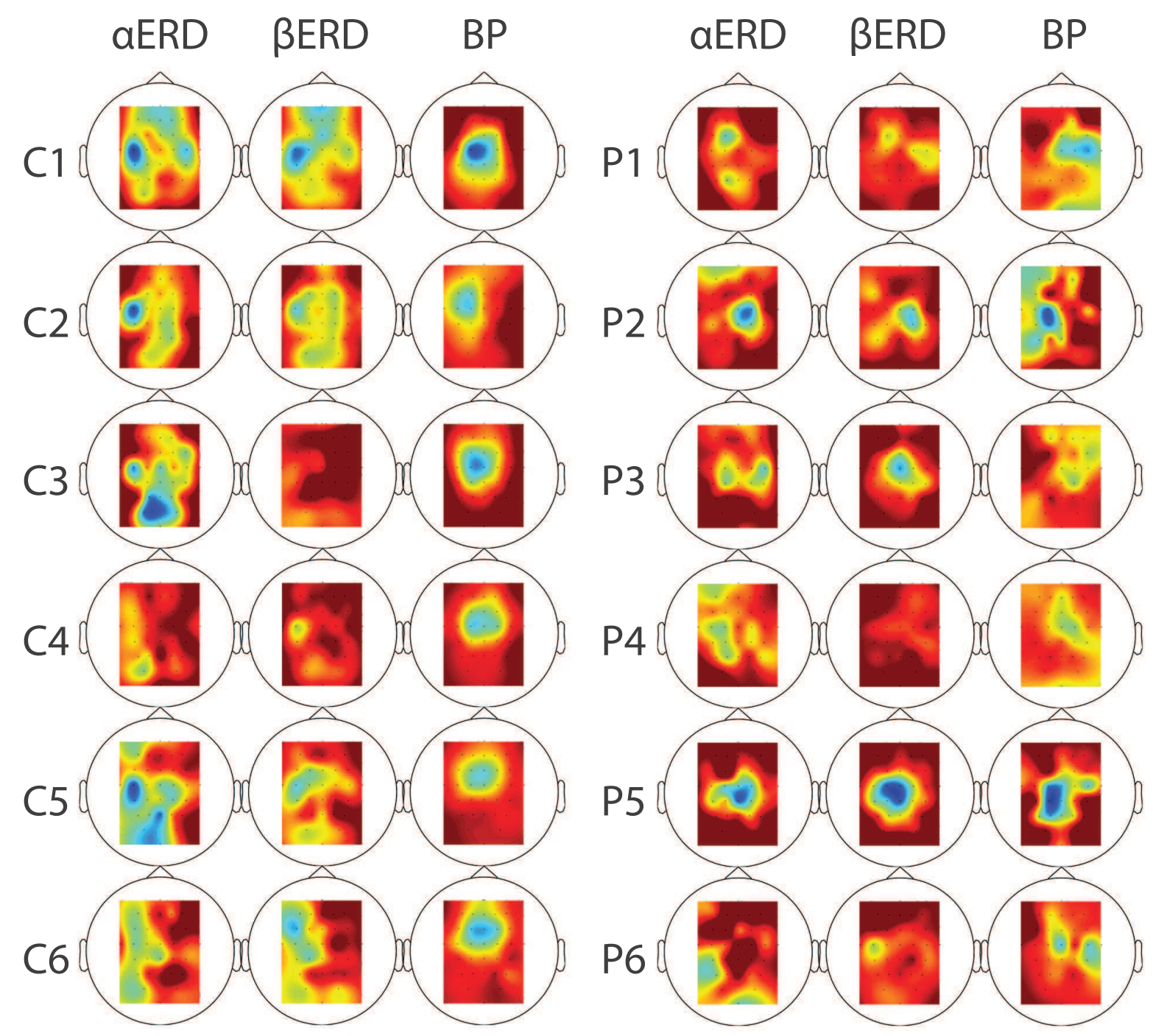

Figure 2. ERD and BP spatial maps with healthy subjects (left) and patients (right). Left and central columns show the spatial distribution of the $\alpha$-ERD and $\beta$-ERD (normalized power changes) obtained by comparing a window of $1.5 \mathrm{~s}$ ending at the movement onset with an equivalent window $4 \mathrm{~s}$ before the onset. The third column shows the spatial distribution of the BP peak amplitude. For each column, the same colour scales are used with all subjects. Colour scale normalization is performed representing the lowest value in each column with dark blue and calibrating the level of dark red in order to optimize the patterns representation.

a more spatially spread distribution of selected features is obtained. Features from the midline (around $\mathrm{Cz}$ ) become more relevant in this case. The selection of features from the alpha or beta band varies for each subject, although beta band features predominate. Finally, the tables show that selected features relative to the alpha-band in the case of the patients present lower frequencies than the ones in the group of healthy subjects.

Fig. 4 compares the detection results obtained with the combined detector (ERD and $\mathrm{BP}$ ) with the results obtained by detectors based only on the BP or the ERD. Statistically significant differences between the three detectors are found in GT, TP 


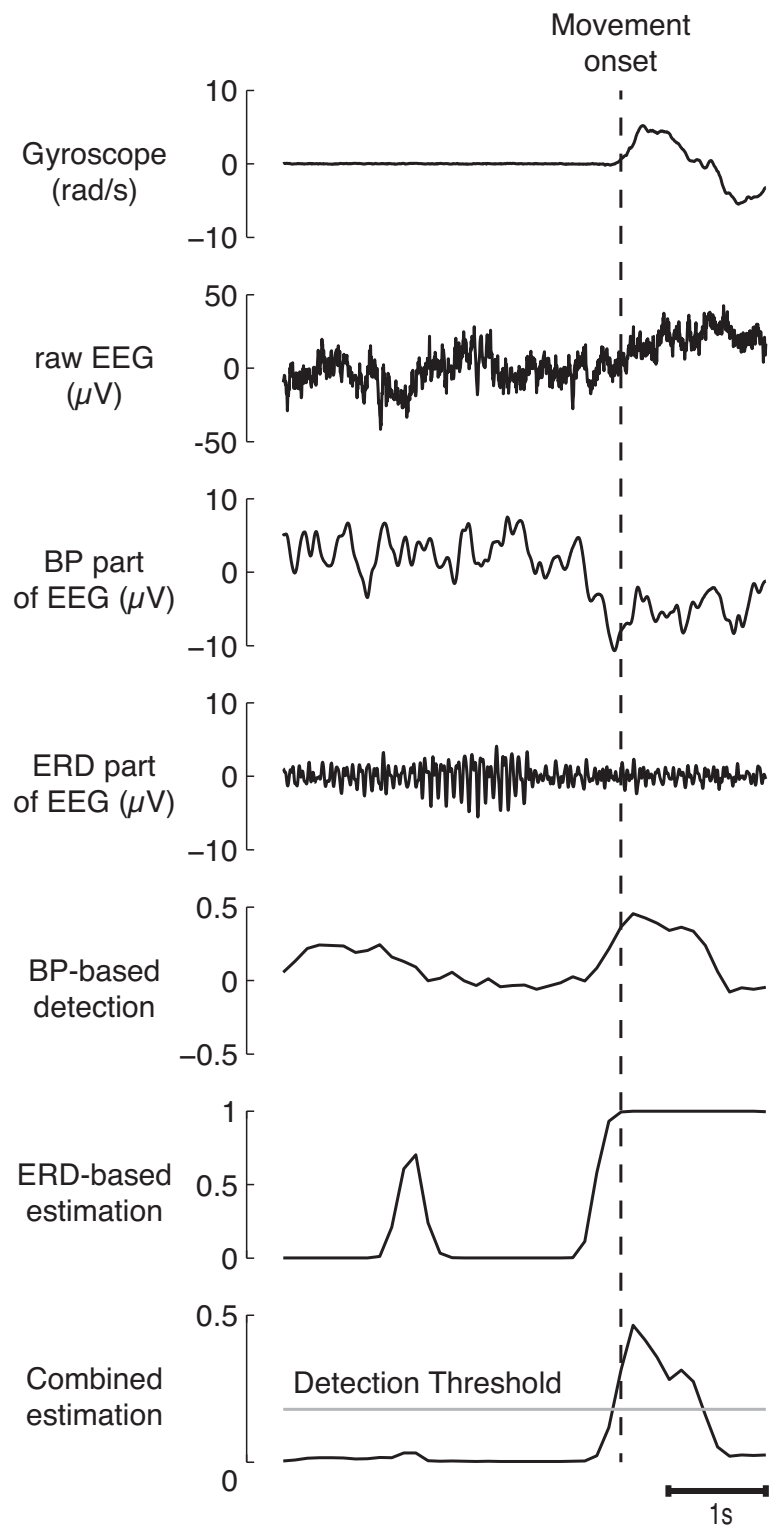

Figure 3. Simulated online function of the single-trial EEG-based detector of onsets of voluntary movements. The plots show from top to bottom: 1) the gyroscopic data used to locate the actual onset of the movement, 2) the raw EEG signal of a single channel, 3) the virtual channel obtained after spatial and temporal filtering the EEG signal to detect the BP pattern, 4) the EEG signal in one channel after applying a small laplacian filter and a band-pass filter (between $6 \mathrm{~Hz}$ and $35 \mathrm{~Hz}$ ) for the ERD-based detection, 5) the output of the matched filter applied by the BP-based detector, 6) the output of the bayesian classifier applied by the ERD-based detector, and 7) the final estimation of the intention to move and the optimal threshold level used to convert the estimation to a boolean signal.

and $\mathrm{FP} / \min (p=0.002, p=0.010$ and $p=0.008$, respectively). Pos-hoc multiple comparisons show significant differences between the ERD-based detector and the combined detector in GT $(p=0.007)$ and $\mathrm{FP} / \mathrm{min}(p=0.015)$, but not in $\mathrm{TP}(p=$ 0.192). In the comparison between the BP-based detector and the combined detector, 


\begin{tabular}{ccccc} 
Code & GoodTr $(\%)$ & $\mathrm{TP}(\%)$ & $\mathrm{FP} / \mathrm{min}$ & Latency $(\mathrm{ms})$ \\
\hline C1 & 81.3 & 82.8 & 0.47 & $-48 \pm 351$ \\
C2 & 63.8 & 81.0 & 1.34 & $-24 \pm 278$ \\
C3 & 39.0 & 56.1 & 2.63 & $-180 \pm 476$ \\
C4 & 64.6 & 70.8 & 0.38 & $-198 \pm 322$ \\
C5 & 69.8 & 84.9 & 1.13 & $-3 \pm 388$ \\
C6 & 61.5 & 71.2 & 1.96 & $-164 \pm 290$ \\
\hline Average & $63.3 \pm 13.8$ & $74.5 \pm 10.8$ & $1.32 \pm 0.87$ & $-89.9 \pm 349.2$ \\
\hline P1 & 56.5 & 84.8 & 1.83 & $-58 \pm 368$ \\
P2 & 75.0 & 83.3 & 0.92 & $123 \pm 290$ \\
P3 & 60.3 & 80.9 & 1.94 & $98 \pm 386$ \\
P4 & 60.0 & 70.0 & 1.08 & $83 \pm 449$ \\
P5 & 100.0 & 100.0 & 0.00 & $-89 \pm 147$ \\
P6 & 46.5 & 74.4 & 3.21 & $50 \pm 520$ \\
\hline Average & $66.4 \pm 18.8$ & $82.2 \pm 10.4$ & $1.50 \pm 1.09$ & $35.9 \pm 352.3$
\end{tabular}

Table 2. Detection results obtained with control subjects and patients

\begin{tabular}{cccccc}
$\mathrm{C} 1$ & $\mathrm{C} 2$ & $\mathrm{C} 3$ & $\mathrm{C} 4$ & $\mathrm{C} 5$ & $\mathrm{C} 6$ \\
\hline $\mathrm{C} 3 / 21 \mathrm{~Hz}$ & $\mathrm{C} 3 / 12 \mathrm{~Hz}$ & $\mathrm{Pz} / 12 \mathrm{~Hz}$ & $\mathrm{~F} 1 / 7 \mathrm{~Hz}$ & $\mathrm{C} 3 / 12 \mathrm{~Hz}$ & $\mathrm{FC} 3 / 19 \mathrm{~Hz}$ \\
$\mathrm{CP} 3 / 21 \mathrm{~Hz}$ & $\mathrm{C} 3 / 11 \mathrm{~Hz}$ & $\mathrm{C} 3 / 12 \mathrm{~Hz}$ & $\mathrm{~F} 1 / 8 \mathrm{~Hz}$ & $\mathrm{C} 3 / 19 \mathrm{~Hz}$ & $\mathrm{CP} 1 / 19 \mathrm{~Hz}$ \\
$\mathrm{C} 3 / 20 \mathrm{~Hz}$ & $\mathrm{C} 3 / 23 \mathrm{~Hz}$ & $\mathrm{C} 3 / 13 \mathrm{~Hz}$ & $\mathrm{C} 6 / 29 \mathrm{~Hz}$ & $\mathrm{C} 3 / 11 \mathrm{~Hz}$ & $\mathrm{FC} 3 / 20 \mathrm{~Hz}$ \\
$\mathrm{CP} 3 / 20 \mathrm{~Hz}$ & $\mathrm{FC} 1 / 18 \mathrm{~Hz}$ & $\mathrm{FC} 4 / 9 \mathrm{~Hz}$ & $\mathrm{C} 3 / 27 \mathrm{~Hz}$ & $\mathrm{CP} 3 / 10 \mathrm{~Hz}$ & $\mathrm{FC} 3 / 18 \mathrm{~Hz}$ \\
$\mathrm{C} 3 / 10 \mathrm{~Hz}$ & $\mathrm{FC} 1 / 17 \mathrm{~Hz}$ & $\mathrm{P} 1 / 12 \mathrm{~Hz}$ & $\mathrm{FC} 1 / 23 \mathrm{~Hz}$ & $\mathrm{CP} 3 / 11 \mathrm{~Hz}$ & $\mathrm{~F} 3 / 19 \mathrm{~Hz}$ \\
$\mathrm{C} 3 / 19 \mathrm{~Hz}$ & $\mathrm{C} 3 / 22 \mathrm{~Hz}$ & $\mathrm{P} 1 / 11 \mathrm{~Hz}$ & $\mathrm{C} 3 / 26 \mathrm{~Hz}$ & $\mathrm{C} 3 / 22 \mathrm{~Hz}$ & $\mathrm{CPz} / 20 \mathrm{~Hz}$ \\
$\mathrm{C} 3 / 22 \mathrm{~Hz}$ & $\mathrm{C} 2 / 17 \mathrm{~Hz}$ & $\mathrm{CP} 1 / 8 \mathrm{~Hz}$ & $\mathrm{C} 3 / 24 \mathrm{~Hz}$ & $\mathrm{CP} 3 / 12 \mathrm{~Hz}$ & $\mathrm{C} 1 / 19 \mathrm{~Hz}$ \\
$\mathrm{CP} 3 / 19 \mathrm{~Hz}$ & $\mathrm{FC} 1 / 19 \mathrm{~Hz}$ & $\mathrm{Pz} / 10 \mathrm{~Hz}$ & $\mathrm{C} 3 / 28 \mathrm{~Hz}$ & $\mathrm{Pz} / 11 \mathrm{~Hz}$ & $\mathrm{~F} 3 / 18 \mathrm{~Hz}$ \\
$\mathrm{C} 3 / 9 \mathrm{~Hz}$ & $\mathrm{FC} 1 / 14 \mathrm{~Hz}$ & $\mathrm{P} 1 / 9 \mathrm{~Hz}$ & $\mathrm{FC} 2 / 18 \mathrm{~Hz}$ & $\mathrm{C} 3 / 18 \mathrm{~Hz}$ & $\mathrm{CP} 3 / 18 \mathrm{~Hz}$ \\
$\mathrm{CP} 3 / 22 \mathrm{~Hz}$ & $\mathrm{C} 3 / 13 \mathrm{~Hz}$ & $\mathrm{FC} 4 / 10 \mathrm{~Hz}$ & $\mathrm{C} 3 / 29 \mathrm{~Hz}$ & $\mathrm{CP} 3 / 13 \mathrm{~Hz}$ & $\mathrm{FC} 3 / 17 \mathrm{~Hz}$
\end{tabular}

Table 3. Features selected by the ERD-based detector for the control group

\begin{tabular}{cccccc}
$\mathrm{P} 1$ & $\mathrm{P} 2$ & $\mathrm{P} 3$ & $\mathrm{P} 4$ & $\mathrm{P} 5$ & $\mathrm{P} 6$ \\
\hline $\mathrm{C} 1 / 9 \mathrm{~Hz}$ & $\mathrm{C} 2 / 9 \mathrm{~Hz}$ & $\mathrm{Cz} / 20 \mathrm{~Hz}$ & $\mathrm{~F} 3 / 8 \mathrm{~Hz}$ & $\mathrm{CP} 2 / 13 \mathrm{~Hz}$ & $\mathrm{C} 3 / 14 \mathrm{~Hz}$ \\
$\mathrm{Cz} / 13 \mathrm{~Hz}$ & $\mathrm{C} 2 / 8 \mathrm{~Hz}$ & $\mathrm{Cz} / 21 \mathrm{~Hz}$ & $\mathrm{C} 1 / 10 \mathrm{~Hz}$ & $\mathrm{C} 2 / 13 \mathrm{~Hz}$ & $\mathrm{P} 2 / 18 \mathrm{~Hz}$ \\
$\mathrm{FC} 1 / 10 \mathrm{~Hz}$ & $\mathrm{C} 2 / 10 \mathrm{~Hz}$ & $\mathrm{Cz} / 13 \mathrm{~Hz}$ & $\mathrm{~F} 3 / 9 \mathrm{~Hz}$ & $\mathrm{C} 1 / 22 \mathrm{~Hz}$ & $\mathrm{C} 3 / 19 \mathrm{~Hz}$ \\
$\mathrm{FC} 1 / 13 \mathrm{~Hz}$ & $\mathrm{CP} 2 / 18 \mathrm{~Hz}$ & $\mathrm{Cz} / 22 \mathrm{~Hz}$ & $\mathrm{C} 2 / 11 \mathrm{~Hz}$ & $\mathrm{C} 1 / 21 \mathrm{~Hz}$ & $\mathrm{C} 2 / 23 \mathrm{~Hz}$ \\
$\mathrm{C} 1 / 10 \mathrm{~Hz}$ & $\mathrm{C} 2 / 7 \mathrm{~Hz}$ & $\mathrm{Cz} / 14 \mathrm{~Hz}$ & $\mathrm{~F} 1 / 8 \mathrm{~Hz}$ & $\mathrm{Cz} / 21 \mathrm{~Hz}$ & $\mathrm{CP} 3 / 14 \mathrm{~Hz}$ \\
$\mathrm{CP} 4 / 18 \mathrm{~Hz}$ & $\mathrm{Cz} / 9 \mathrm{~Hz}$ & $\mathrm{Cz} / 16 \mathrm{~Hz}$ & $\mathrm{P} 1 / 10 \mathrm{~Hz}$ & $\mathrm{C} 1 / 20 \mathrm{~Hz}$ & $\mathrm{CP} 1 / 15 \mathrm{~Hz}$ \\
$\mathrm{FC} 1 / 9 \mathrm{~Hz}$ & $\mathrm{Cz} / 10 \mathrm{~Hz}$ & $\mathrm{Cz} / 15 \mathrm{~Hz}$ & $\mathrm{C} 1 / 9 \mathrm{~Hz}$ & $\mathrm{CPz} / 22 \mathrm{~Hz}$ & $\mathrm{FC} 2 / 19 \mathrm{~Hz}$ \\
$\mathrm{FC} 1 / 11 \mathrm{~Hz}$ & $\mathrm{CP} 2 / 19 \mathrm{~Hz}$ & $\mathrm{Cz} / 17 \mathrm{~Hz}$ & $\mathrm{P} 3 / 8 \mathrm{~Hz}$ & $\mathrm{CPz} / 16 \mathrm{~Hz}$ & $\mathrm{Pz} / 22 \mathrm{~Hz}$ \\
$\mathrm{C} 1 / 12 \mathrm{~Hz}$ & $\mathrm{CP} 2 / 17 \mathrm{~Hz}$ & $\mathrm{CP} 1 / 11 \mathrm{~Hz}$ & $\mathrm{~F} 4 / 20 \mathrm{~Hz}$ & $\mathrm{CP} / 12 \mathrm{~Hz}$ & $\mathrm{CP} 4 / 21 \mathrm{~Hz}$ \\
$\mathrm{C} 1 / 13 \mathrm{~Hz}$ & $\mathrm{Cz} / 8 \mathrm{~Hz}$ & $\mathrm{Cz} / 19 \mathrm{~Hz}$ & $\mathrm{FC} 3 / 8 \mathrm{~Hz}$ & $\mathrm{C} 1 / 23 \mathrm{~Hz}$ & $\mathrm{CP} 3 / 11 \mathrm{~Hz}$
\end{tabular}

Table 4. Features selected by the ERD-based detector for the patients

significant differences are found in GT $(p=0.003)$ and $\operatorname{TP}(p=0.003)$, but not in $\mathrm{FP} / \min (p=0.0 .059)$. Finally, no significant differences are found in GT $(p=0.611)$, $\mathrm{TP}(p=1)$ and $\mathrm{FP} / \mathrm{min}(p=0.305)$ between the detector based on the ERD and the one based on the BP. 
For healthy subjects, the detector combining ERD and BP information achieves 6.5 $\pm 5.2 \%$ more GT than the BP-based detector and $22.4 \pm 10.0 \%$ more GT than the ERD-based detector (see Table 5). For patients, the percentage of GT also increases when using the combined detector $(13.3 \pm 10.9 \%$ and $12.6 \pm 16.3 \%$ increase as compared with the BP- and ERD-based detectors, respectively).
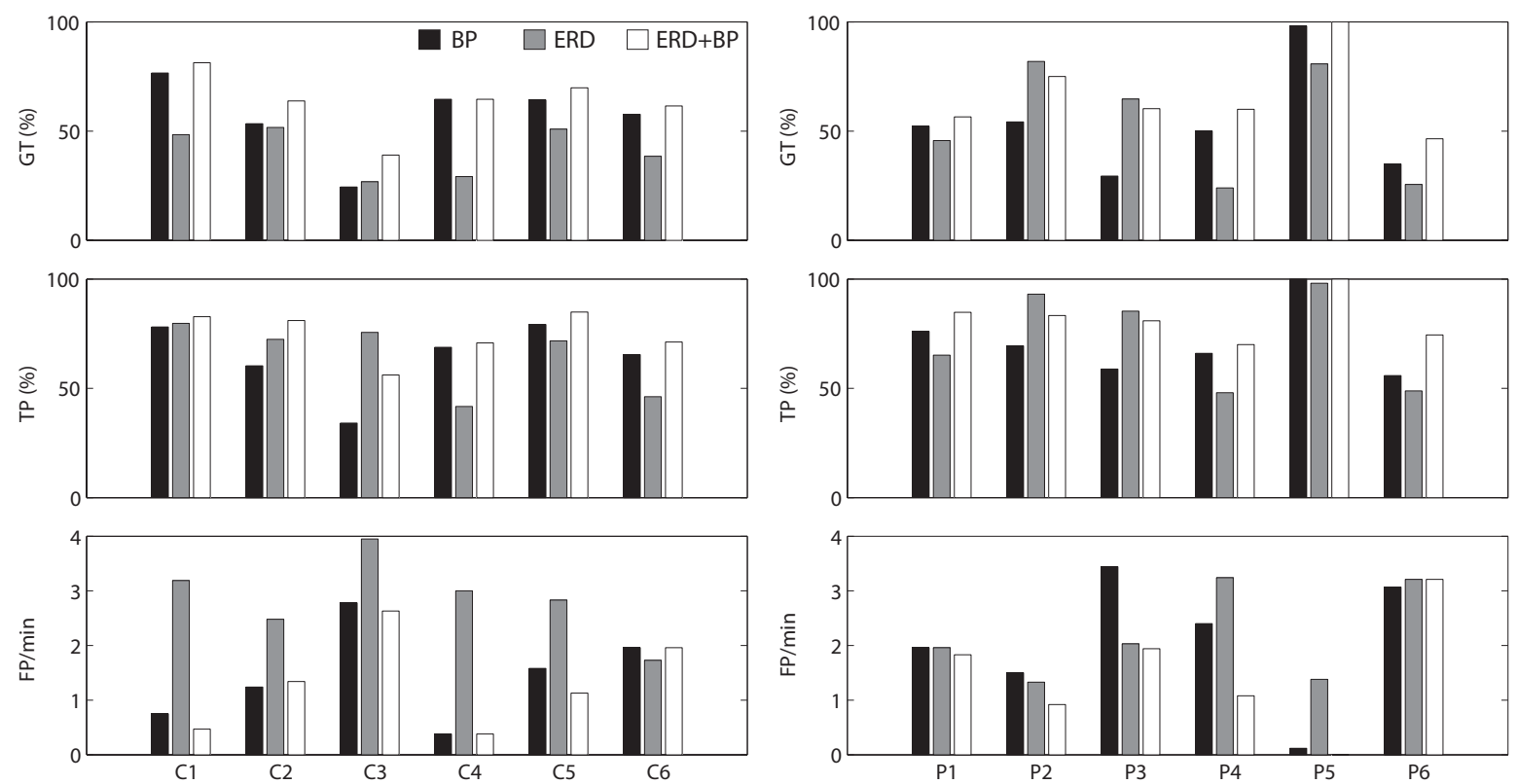

Figure 4. Performances of the three compared detectors (BP-based, ERD-based and combined detector) in the healthy subjects group (left) and in the patients (right) in terms of $\mathrm{GT}$, TP and $\mathrm{FP} / \mathrm{min}$

\begin{tabular}{ccc} 
Code & GT (\%) vs BP & GT (\%) vs ERD \\
\hline C1 & 4.7 & 32.8 \\
C2 & 10.3 & 12.1 \\
C3 & 14.6 & 12.2 \\
C4 & 0.0 & 35.4 \\
C5 & 5.7 & 18.9 \\
C6 & 3.8 & 23.1 \\
\hline Average & $6.5 \pm 5.2$ & $22.4 \pm 10.0$ \\
\hline P1 & 4.3 & 10.9 \\
P2 & 20.8 & -6.9 \\
P3 & 30.9 & -4.4 \\
P4 & 10.0 & 36.0 \\
P5 & 1.9 & 19.2 \\
P6 & 11.6 & 20.9 \\
\hline Average & $13.3 \pm 10.9$ & $12.6 \pm 16.3$
\end{tabular}

Table 5. Gain in the performance of the detector (GT) when using the combined information of the ERD and BP compared to the use of either of these patterns alone.

The latencies in the detections of the movement onsets are represented by means of histograms in Fig. 5. The latencies obtained when using the detectors based only on the $\mathrm{BP}$ or the ERD information are superimposed in the figure. The histograms shown 
depend on how much do the ERD and BP patterns vary across trials with respect to the onsets of the movements, and also on the detection threshold applied to each one of the three detectors. The figure shows a more delayed distribution of the detections with the group of patients. Nonetheless, around $85 \%$ of these BP detections are located earlier than +375 ms. Given that the window used for the BP detector are $1.5 \mathrm{~s}$ long, this result supports the absence of movement artefacts in the activity analyzed. The ERD-based detector appears to be the less precise in terms of latencies of the detections, while the BP-based detector presents distributions clearly centred at $\mathrm{t}=0 \mathrm{~s}$. Also noticeably, the ERD-based detector shows a certain degree of anticipation in the detections of movement onsets in the group of healthy subjects, although it generates delayed detections in the case of the patients.
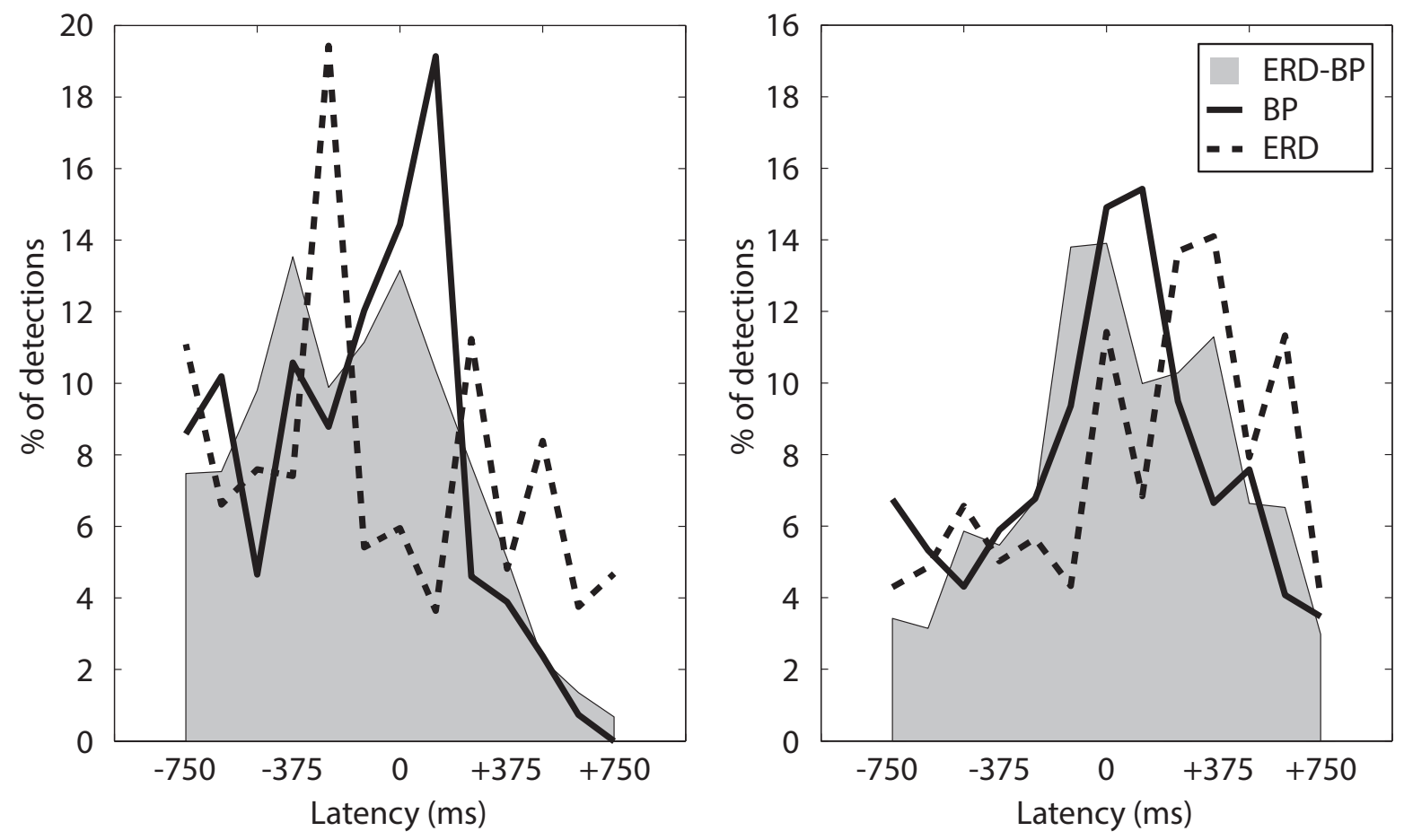

Figure 5. Histograms of the distances between the movement detections and the actual movement onsets for healthy subjects (left panel) and stroke patients (right panel). The histograms of the detectors based only on the ERD or the BP are superimposed in the graphs

\section{Discussion}

The accuracy with which movements can be detected online by using EEG activity (both in terms of temporal precision and ratio between true and false activations) represents an important criterion when deciding whether BCI technology can be brought to clinical practice in neurorehabilitation environments. This study showed the results of an EEGbased detector of voluntary movement onsets that combined information extracted from the processing of cortical rhythms and slow cortical potentials. This is the first time 
that both sources of information have been combined to this end. It is also the first time the benefits of a detector combining information from the ERD and BP patterns in patients with stroke have been demonstrated.

Previous studies have described several aspects on the characterization of the BP to locate movement onsets. Garipelli et al. studied the relevance of choosing appropriate spatial and temporal filters to extract the BP pattern [21], without showing results regarding temporal precision in the detections. In a study by Lew et al., average results of BP detection were presented for healthy subjects and stroke patients, although no single trial validation was carried out [22]. To the authors' knowledge, no one thus far has studied the detection of upper-limb voluntary movements based on the detection of the $\mathrm{BP}$ and using an online feasible design.

In a recent study, $\mathrm{Xu}$ et al. presented a system using a manifold method (Locality Preserving Projection) with a LDA classifier to optimize the classification of the BP. The algorithm was tested on healthy subjects performing ankle dorsiflexions. The TP and $\mathrm{FP} /$ min results obtained in that study (79 $\pm 12 \%$ and $1.04 \pm 0.8$, respectively) were similar to the ones obtained here with healthy subjects and upper-limb movements. Nevertheless, the average latencies presented in their study ( $315 \pm 165 \mathrm{~ms})$ were higher than the ones obtained here. This differences could be due to variations in the way subjects performed the task in each experiment (differences between upper-limb and lower limb cortical patterns, length of the resting intervals between movements and speed of movements among others). The observed differences could also be due to the combined use of the ERD and BP features proposed here, which enables reduction of the rate of $\mathrm{FP}$ and, as a consequence, enables the selection of less restrictive (more anticipative) detection thresholds.

Although several previous studies have made use of cortical rhythms to either detect movement events $[7,35]$ or to anticipate movement intentions [12,31], no studies so far have tried to use ERD information to locate voluntary movement onsets with time precision. In a previous study by Fatourechi et al., the combined use of cortical rhythms and slow cortical potentials was proposed for an asynchronous BCI, although in that case the device was not intended to detect the onset of voluntary movements [28]. The naïve Bayes classifier described here has demonstrated that the ERD supplies valuable information in this sense. Indeed, we have shown here the benefits of the combined use of the information about the ERD and BP as compared to detectors relying solely on either the BP or the ERD.

Significantly better performances could be achieved with the combined detector in all metrics analysed: a higher number of GT and TP was achieved with lower rates of false activations during the resting intervals. Previous studies have demonstrated that different neural mechanisms are involved in the generation of the ERD and the BP and therefore may justify their complementarity. The BP is assumed to originate in the presupplementary and supplementary motor areas [25,37], which are associated with the movement planning and with the process of attending the intention to move [38]. In contrast, the ERD is first visible over the contralateral motor cortex [4], and it is 
associated with the formation of more specific neural assemblies synchronized at higher frequencies to generate the desired descending motor commands [4,39]. The spatial distribution of both phenomena in the here presented data also points to different cortical sources. Given these evidences, it seems reasonable to point to an improved outcome in the combination of both sources of information to estimate certain aspects regarding motor planning.

Differences in the average ERD and BP patterns between patients and healthy subjects were found. A delayed peak of the BP was observed in the patients group, likely associated with the higher cognitive motor planning time and the slower speed with which stroke patients perform voluntary movements $[17,26]$. Differences in the spatial distribution of both ERD and BP patterns were also observed (see Fig. 2), reflecting altered cortical activation patterns in stroke patients, as described in previous studies $[26,32,40,41]$.

Regarding the single-trial detection results, previous offline studies [13] showed differences in BP-based detection performance with healthy subjects and stroke patients (significantly worse TP results were obtained with the patients). In contrast, the detection results obtained here with the proposed system (in terms of GT, TP and $\mathrm{FP} / \mathrm{min}$ ) were similar for patients and healthy subjects. Apart from the differences in the recruited subjects and paradigms used in both experiments, these better results with patients were likely due to the improved detector performance when the ERD information was used. Specifically, there was a $13.3 \pm 10.9 \%$ increase in numbers of GT as compared to the BP-based detector alone (see Table 5). In addition, differences were observed in the detection latencies: detections in patients were achieved later than with the healthy subjects. According to Fig. 5, this is especially evident in the ERDbased detection (whereas ERD-based detections in healthy subjects tend to anticipate the actual movement onsets, the reverse effect is observed in the group of patients). Such difference may be the combined result of the altered ERD in stroke patients $[32,41]$ and an aging factor [42].

Given the detector design proposed here, the influence of movement artifacts in the detections achieved after the onset of movements are considered negligible. First, regarding the ERD-based system, the combined use of a small laplacian filter and a band-pass filter discarded the presence of movement-related common low-frequency components in the analysed EEG. In addition, the use of premovement signals in the training stage ensured that the Bayesian classifier focused specifically on the ERD phenomenon, as can be seen by analysing the features selected by the Bayesian classifier (see Tables 3 and 4 ).

In the case of BP-based detection, use of spatial filtering together with the spatial distribution of this pattern (see Fig. 2) reduces the chance that artifactual sources are having any influence. Indeed, around $95 \%$ of the detections in the case of the healthy subjects (around $85 \%$ with the patients) were obtained with latencies under $+375 \mathrm{~ms}$ (see Fig. 5). Because a matched filter of $1.5 \mathrm{~s}$ was used, it is highly unlikely that any of these detections were caused by the effect of movement artifacts. In fact, BP-based 
detections later than $+375 \mathrm{~ms}$ in the stroke patients are likely related to the intrinsic difficulties in detectinh the real onsets of the movements and also to the delayed BP observed in these patients due to slower movement velocities with the affected limb [17] and to an increased cognitive motor planning time [26].

Developing EEG-based systems that can be trained in a short period of time is a critical aspect in bringing this technology into clinical practice. The training procedure proposed here assumes that a number of self-initiated movements are performed in the beginning of each session and are used to train the detector (this process takes around $5 \mathrm{~min}$ if 30 movements are used to train the system). In this regard, several studies have proposed methods for using training data from different sessions to calibrate the BCI system $[43,44]$, and these methods may be considered in future studies regarding the EEG-based detector proposed here.

Finally, gyroscopic data were used to locate the movement events to extract and characterize the subject-specific ERD and BP patterns. Similar previous studies have frequently used muscle activation data (from EMG) for such purposes. In this case, because functional upper limb movements were measured on stroke patients, detecting the onsets of the movements from muscle activation became difficult, particularly in the patients with muscle spasticity. On the contrary, by using kinematic data from the upper-limb segments, it becomes possible to finely detect when a functional movement starts without significant latencies, considering that the electromechanical delay for upper-limb movements is relatively small (on the order of tens of milliseconds [45]). In agreement with this observation, results presented here of average BP patterns in healthy subjects and patients -obtained with movement references based on gyroscopic data- show peaks of the BP with similar latencies than those observed in other studies using EMG data and healthy subjects [19].

\section{Conclusion}

EEG-based detection of the onsets of voluntary upper-limb movements combining information about cortical rhythms and slow cortical potentials has been proposed and tested in healthy subjects and stroke patients. With the proposed system the average number of true positives $(74.5 \pm 13.8 \%)$ and false activations per minute $(1.32 \pm 0.87)$ obtained in the group of healthy subjects was similar to comparable previous studies, and the latencies of the detections were smaller here $(-89.9 \pm 349.2 \mathrm{~ms})$. Remarkably, similar results to those obtained with the healthy subjects were observed in the group of patients (true positives $=82.2 \pm 10.4 \%$; FP/min $=1.50 \pm 1.09$ ), although in this case, higher latencies in the detections were observed (35.9 \pm 352.3$)$. By comparing the proposed detector with two alternatives relying either on the ERD or the BP patterns, the study demonstrated the importance of combining these two sources of information to boost the performance of the movement onset detector. 


\section{Acknowledgments}

This work has been funded by grant from the Spanish Ministry of Science and Innovation CONSOLIDER INGENIO, project HYPER (Hybrid NeuroProsthetic and NeuroRobotic Devices for Functional Compensation and Rehabilitation of Motor Disorders, CSD200900067), from Proyectos Cero of FGCSIC, Obra Social la Caixa, CSIC, and from the project PIE 201050E087. 
[1] G. Chatrian, M. Petersen, and J. Lazarte, "The blocking of the rolandic wicket rhythm and some central changes related to movement," Electroencephalography and Clinical Neurophysiology, vol. 11, no. 3, pp. 497-510, 1959.

[2] B. Libet, E. Wright, and C. Gleason, "Readiness-potentials preceding unrestricted 'spontaneous' vs. pre-planned voluntary acts," Electroencephalography and Clinical Neurophysiology, vol. 54, no. 3, pp. 322-335, Sep. 1982.

[3] G. Pfurtscheller, "Central beta rhythm during sensorimotor activities in man," Electroencephalography and Clinical Neurophysiology, vol. 51, no. 3, pp. 253-264, Mar. 1981.

[4] G. Pfurtscheller and F. H. L. da Silva, "Event-related EEG/EMG Synchronization and Desynchronization: Basic Principles," Clinical Neurophysiology, vol. 110, pp. 1842-1857, 1999.

[5] F. Galán, M. Nuttin, E. Lew, P. W. Ferrez, G. Vanacker, J. Philips, and J. R. Millán, "A brainactuated wheelchair: asynchronous and non-invasive Brain-computer interfaces for continuous control of robots," Clinical Neurophysiology, vol. 119, no. 9, pp. 2159-69, Sep. 2008.

[6] J. R. Wolpaw, D. J. McFarland, T. M. Vaughan, and G. Schalk, "The Wadsworth Center braincomputer interface (BCI) research and development program." IEEE transactions on neural systems and rehabilitation engineering, vol. 11, no. 2, pp. 204-7, Jun. 2003.

[7] G. Müller-Putz, R. Scherer, G. Pfurtscheller, and R. Rupp, "EEG-based neuroprosthesis control: a step towards clinical practice." Neuroscience letters, vol. 382, no. 1-2, pp. 169-74, 2005.

[8] F. Nijboer, E. W. Sellers, J. Mellinger, M. a. Jordan, T. Matuz, A. Furdea, S. Halder, U. Mochty, D. J. Krusienski, T. M. Vaughan, J. R. Wolpaw, N. Birbaumer, and A. Kübler, "A P300-based brain-computer interface for people with amyotrophic lateral sclerosis." Clinical neurophysiology, vol. 119, no. 8, pp. 1909-16, Aug. 2008.

[9] J. J. Daly and J. R. Wolpaw, "Brain-computer interfaces in neurological rehabilitation," The Lancet Neurology, vol. 7, no. 11, pp. 1032-1043, Nov. 2008.

[10] E. Buch, C. Weber, L. G. Cohen, C. Braun, M. A. Dimyan, T. Ard, J. Mellinger, A. Caria, S. Soekadar, A. Fourkas, and N. Birbaumer, "Think to move: a neuromagnetic brain-computer interface (BCI) system for chronic stroke." Stroke, vol. 39, no. 3, pp. 910-7, Mar. 2008.

[11] G. Pfurtscheller and T. Solis-Escalante, "Could the beta rebound in the EEG be suitable to realize a "brain switch"?" Clinical Neurophysiology, vol. 120, no. 1, pp. 24-9, Jan. 2009.

[12] O. Bai, V. Rathi, P. Lin, D. Huang, H. Battapady, D.-Y. Fei, L. Schneider, E. Houdayer, $\mathrm{X}$. Chen, and M. Hallett, "Prediction of human voluntary movement before it occurs," Clinical Neurophysiology, vol. 122(2), pp. 364-72, Jul. 2011.

[13] I. K. Niazi, N. Jiang, O. Tiberghien, J. r. F. k. Nielsen, K. Dremstrup, and D. Farina, "Detection of movement intention from single-trial movement-related cortical potentials." Journal of neural engineering, vol. 8, no. 6, p. 066009, Dec. 2011.

[14] G. Pfurtscheller, C. Brunner, A. Schlögl, F. H. L. da Silva, and F. H. Lopes da Silva, "Mu rhythm (de)synchronization and EEG single-trial classification of different motor imagery tasks," NeuroImage, vol. 31, no. 1, pp. 153-9, May 2006.

[15] V. Morash, O. Bai, S. Furlani, P. Lin, and M. Hallett, "Classifying EEG signals preceding right hand, left hand, tongue, and right foot movements and motor imageries," Clinical Neurophysiology, vol. 119, no. 11, pp. 2570-8, Nov. 2008.

[16] Y. Gu, K. Dremstrup, and D. Farina, "Single-trial discrimination of type and speed of wrist movements from EEG recordings." Clinical neurophysiology : official journal of the International Federation of Clinical Neurophysiology, vol. 120, no. 8, pp. 1596-600, Aug. 2009.

[17] M. Jochumsen, I. K. Niazi, N. Mrachacz-kersting, D. Farina, and K. Dremstrup, "Detection and classification of movement-related cortical potentials associated with task force and speed." Journal of neural engineering, vol. 10, no. 5, p. 056015, Aug. 2013.

[18] J. A. Gallego, J. Ibáñez, J. L. Dideriksen, J. I. Serrano, M. D. del Castillo, D. Farina, and E. Rocon, "A Multimodal HumanRobot Interface to Drive a Neuroprosthesis for Tremor Management," IEEE Transactions on Systems, Man, and Cybernetics, Part C (Applications and Reviews), vol. 42, no. 6, pp. 1159-1168, Nov. 2012. 
[19] N. Mrachacz-Kersting, S. R. Kristensen, I. K. Niazi, and D. Farina, "Precise temporal association between cortical potentials evoked by motor imagination and afference induces cortical plasticity." The Journal of physiology, vol. 590, no. Pt 7, pp. 1669-82, Apr. 2012.

[20] I. K. Niazi, N. Mrachacz-Kersting, N. Jiang, K. Dremstrup, and D. Farina, "Peripheral electrical stimulation triggered by self-paced detection of motor intention enhances motor evoked potentials." IEEE transactions on neural systems and rehabilitation engineering, vol. 20, no. 4, pp. 595-604, Jul. 2012.

[21] G. Garipelli, R. Chavarriaga, and J. del R Millán, "Single trial analysis of slow cortical potentials: a study on anticipation related potentials," Journal of neural engineering, vol. 10, no. 3, p. 036014, Jun. 2013.

[22] E. Lew, R. Chavarriaga, S. Silvoni, and J. R. Millán, "Detection of self-paced reaching movement intention from EEG signals." Frontiers in neuroengineering, vol. 5, no. July, p. 13, Jan. 2012.

[23] R. Xu, N. Jiang, C. Lin, N. Mrachacz-Kersting, K. Dremstrup, and D. Farina, "Enhanced low-latency detection of motor intention from EEG for closed-loop brain-computer interface applications." IEEE transactions on bio-medical engineering, vol. 61, no. 2, pp. 288-96, Feb. 2014.

[24] H. H. Kornhuber and L. Deecke, "Hirnpotentialänderungen bei Willkürbewegungen und passiven Bewegungen des Menschen: Bereitschaftspotential und reafferente Potentiale," Pflügers Archiv für die Gesamte Physiologie des Menschen und der Tiere, vol. 284, no. 1, pp. 1-17, 1965.

[25] H. Shibasaki and M. Hallett, "What is the Bereitschaftspotential?" Clinical neurophysiology, vol. 117, no. 11, pp. 2341-56, Nov. 2006.

[26] J. J. Daly, Y. Fang, E. M. Perepezko, V. Siemionow, and G. H. Yue, "Prolonged Cognitive Planning Time, Elevated Cognitive Effort, and Relationship to Coordination and Motor," Rehabilitation, vol. 14, no. 2, pp. 168-171, 2006.

[27] Y. Fang, G. H. Yue, K. Hrovat, V. Sahgal, and J. J. Daly, "Abnormal cognitive planning and movement smoothness control for a complex shoulder/elbow motor task in stroke survivors." Journal of the neurological sciences, vol. 256, no. 1-2, pp. 21-9, May 2007.

[28] M. Fatourechi, R. K. Ward, and G. E. Birch, "A self-paced brain-computer interface system with a low false positive rate." Journal of neural engineering, vol. 5, no. 1, pp. 9-23, Mar. 2008.

[29] H. Jasper and W. Penfield, "Electrocorticograms in man: Effect of voluntary movement upon the electrical activity of the precentral gyrus," Archiv for Psychiatrie und Nervenkrankheiten, vol. 183, no. 1-2, pp. 163-174, 1949.

[30] O. Bai, Z. Mari, S. Vorbach, and M. Hallett, "Asymmetric Spatiotemporal Patterns of Eventrelated Desynchronization preceding Voluntary Sequential Finger Movements: a High-resolution EEG Study," Clinical Neurophysiology, vol. 116, pp. 1213-1221, 2005.

[31] J. Ibáñez, J. Serrano, M. del Castillo, J. A. Gallego, and E. Rocon, "Online detector of movement intention based on EEG - Application in tremor patients," Biomedical Signal Processing and Control, vol. 8, no. 6, pp. 822-829, Nov. 2013.

[32] M. Stepien, J. Conradi, G. Waterstraat, F. U. Hohlefeld, G. Curio, and V. V. Nikulin, "Eventrelated desynchronization of sensorimotor EEG rhythms in hemiparetic patients with acute stroke." Neuroscience letters, Nov. 2010.

[33] O. Bai, P. Lin, S. Vorbach, J. Li, S. Furlani, and M. Hallett, "Exploration of computational methods for classification of movement intention during human voluntary movement from single trial EEG." Clinical Neurophysiology, vol. 118, no. 12, pp. 2637-55, Dec. 2007.

[34] B. Hjorth, "An on-line transformation of EEG scalp potentials into orthogonal source derivations," Electroencephalogr Clin Neurophysiol, vol. 39, no. 5, pp. 526-30, Dec. 1975.

[35] G. Townsend, B. Grainmann, and G. Pfurtscheller, "Continuous EEG Classification During Motor Imagery-Simulation of an Asynchronous BCI," IEEE Transactions on Neural Systems and Rehabilitation Engineering, vol. 12, no. 2, pp. 258-265, 2004.

[36] S. G. Mason, J. Kronegg, J. Huggins, M. Fatourechi, and A. Schlögl, "Evaluating the performance of self-paced brain computer interface technology," Neil Squire Soc., Vancouver, BC, Canada, 
Tech. Rep, vol. 0, 2006.

[37] C. Babiloni, F. Carducci, F. Cincotti, P. M. Rossini, C. Neuper, G. Pfurtscheller, and F. Babiloni, "Human movement-related potentials vs desynchronization of EEG alpha rhythm: a highresolution EEG study." NeuroImage, vol. 10, no. 6, pp. 658-65, Dec. 1999.

[38] H. C. Lau, R. D. Rogers, P. Haggard, and R. E. Passingham, "Attention to intention." Science (New York, N.Y.), vol. 303, no. 5661, pp. 1208-10, Mar. 2004.

[39] G. Buzsáki and A. Draguhn, "Neuronal oscillations in cortical networks." Science, vol. 304, no. 5679, pp. 1926-9, Jun. 2004.

[40] H. Wiese, P. Stude, K. Nebel, D. Osenberg, V. Völzke, W. Ischebeck, D. Stolke, H. C. Diener, and M. Keidel, "Impaired movement-related potentials in acute frontal traumatic brain injury," Clinical Neurophysiology, vol. 115, pp. 289 - 298, 2004.

[41] T. Platz, "Multimodal EEG analysis in man suggests impairment-specific changes in movementrelated electric brain activity after stroke," Brain, vol. 123, no. 12, pp. 2475-2490, Dec. 2000.

[42] P. Derambure, L. Defebvre, K. Dujardin, J. L. Bourriez, J. M. Jacquesson, A. Destee, and J. D. Guieu, "Effect of aging on the spatio-temporal pattern of event-related desynchronization during a voluntary movement." Electroencephalography and clinical neurophysiology, vol. 89, no. 3, pp. 197-203, Jun. 1993.

[43] P. Shenoy, M. Krauledat, B. Blankertz, R. P. N. Rao, and K. Müller, "Towards adaptive classification for BCI," Journal of Neural Engineering, vol. 3, no. 1, pp. R13-23, Mar. 2006.

[44] I. K. Niazi, N. Jiang, M. Jochumsen, J. r. F. k. Nielsen, K. Dremstrup, and D. Farina, "Detection of movement-related cortical potentials based on subject-independent training." Medical $\mathbb{6}$ biological engineering \& computing, vol. 51, no. 5, pp. 507-12, Jan. 2013.

[45] R. W. Norman and P. V. Komi, "Electromechanical delay in skeletal muscle under normal movement conditions," Acta Physiologica Scandinavica, vol. 106, no. 3, pp. 241-248, Jul. 1979. 